\title{
Sign or Symptom
}

National Cancer Institute

\section{Source}

National Cancer Institute. Sign or Symptom. NCI Thesaurus. Code C100104.

Objective evidence of disease perceptible to the examining practitioner (sign) and subjective evidence of disease perceived by the patient (symptom). 